\title{
Cryptanalysis of a computer cryptography scheme based on a filter bank
}

\author{
David Arroyo ${ }^{\mathrm{a}, *}$, Chengqing $\mathrm{Li}^{\mathrm{b}}$, Shujun $\mathrm{Li}^{\mathrm{c}}$ and \\ Gonzalo Alvarez $^{\text {a }}$ \\ ${ }^{a}$ Instituto de Física Aplicada, Consejo Superior de Investigaciones Científicas, \\ Serrano 144, 28006 Madrid, Spain \\ ${ }^{\mathrm{b}}$ Department of Electronic Engineering, City University of Hong Kong, 83 Tat \\ Chee Avenue, Kowloon Tong, Hong Kong SAR, China \\ ${ }^{\mathrm{c}}$ FernUniversität in Hagen, Lehrgebiet Informationstechnik, Universitätsstraße 2\%, \\ 58084 Hagen, Germany
}

\begin{abstract}
This paper analyzes the security of a recently-proposed signal encryption scheme based on a filter bank. A very critical weakness of this new signal encryption procedure is exploited in order to successfully recover the associated secret key.
\end{abstract}

Key words: Chaotic encryption, logistic map, known-plaintext attack, cryptanalysis

PACS: 05.45.Ac, 47.20.Ky.

\section{Introduction}

The application of chaotic systems to cryptographical issues has been a very important research topic since the 1990s [1-4]. This interest was motivated by the close similarities between some properties of chaotic systems and some characteristics of well-designed cryptosystems [5, Table 1]. Nevertheless, there exist security defects in some chaos-based cryptosystems such that they can be partially or totally broken [6-11].

In [12] the encryption procedure is carried out by decomposing the input plaintext signal into two different subbands and masking each of them with

* Corresponding author: David Arroyo (david.arroyo@iec.csic.es). 
a pseudo-random number sequence generated by iterating the chaotic logistic map. The decomposition of the input plaintext signal $x[n]$ is driven by

$$
\begin{aligned}
& t_{0}[n]=K_{0} \sum_{\forall m} x[m] h_{0}[2 n-m], \\
& t_{1}[n]=K_{1} \sum_{\forall m} x[m] h_{1}[2 n-m],
\end{aligned}
$$

where $h_{0}, h_{1}$ are so-called "analysis filters" and $K_{0}, K_{1}$ are gain factors.

Then, the masking stage generates the ciphertext signal $\left(v_{0}[n], v_{1}[n]\right)$ according to the following equations:

$$
\begin{aligned}
& v_{0}[n]=t_{0}[n]+\alpha_{0}\left(t_{1}[n]\right), \\
& v_{1}[n]=t_{1}[n]-\alpha_{1}\left(v_{0}[n]\right),
\end{aligned}
$$

where $\alpha_{i}(u)=u+s_{i}[n]$ and $s_{i}[n]$ is the state variable of a logistic map with control parameter $\lambda_{i} \in(3,4)$ defined as follow 1

$$
s_{i}[n]=\lambda_{i} s_{i}[n-1]\left(1-s_{i}[n-1]\right) .
$$

Substituting $\alpha_{i}(u)=u+s_{i}[n]$ into Eqs. (3) and (44), we have

$$
\begin{aligned}
& v_{0}[n]=\left(t_{0}[n]+t_{1}[n]\right)+s_{0}[n], \\
& v_{1}[n]=\left(t_{1}[n]-v_{0}[n]\right)-s_{1}[n] .
\end{aligned}
$$

The secret key of the cryptosystem is composed of the initial conditions and the control parameters of the two logistic maps involved, i.e., $s_{0}[0], s_{1}[0], \lambda_{0}$ and $\lambda_{1}$.

The decryption procedure is carried out by doing

$$
\begin{aligned}
& t_{1}[n]=v_{1}[n]+\alpha_{1}\left(v_{0}[n]\right), \\
& t_{0}[n]=v_{0}[n]-\alpha_{0}\left(t_{1}[n]\right) .
\end{aligned}
$$

Then, the plaintext signal is recovered with the following filtering operations:

$$
\tilde{x}[n]=\frac{1}{K_{0}} \sum_{\forall m} t_{0}[m] f_{0}[n-2 m]+\frac{1}{K_{1}} \sum_{\forall m} t_{1}[m] f_{1}[n-2 m],
$$

$\overline{1}$ In [12] the authors use $x_{i}$ to denote the state variable of the logistic map. However, this nomenclature may cause confusion because the plaintext signal is denoted by $x$. Therefore, we turn to use another letter, $s$. In addition, we unify the representation of $x_{i}(k)$ to be in the form $s_{i}[n]$ because all other signals are in the latter form. 


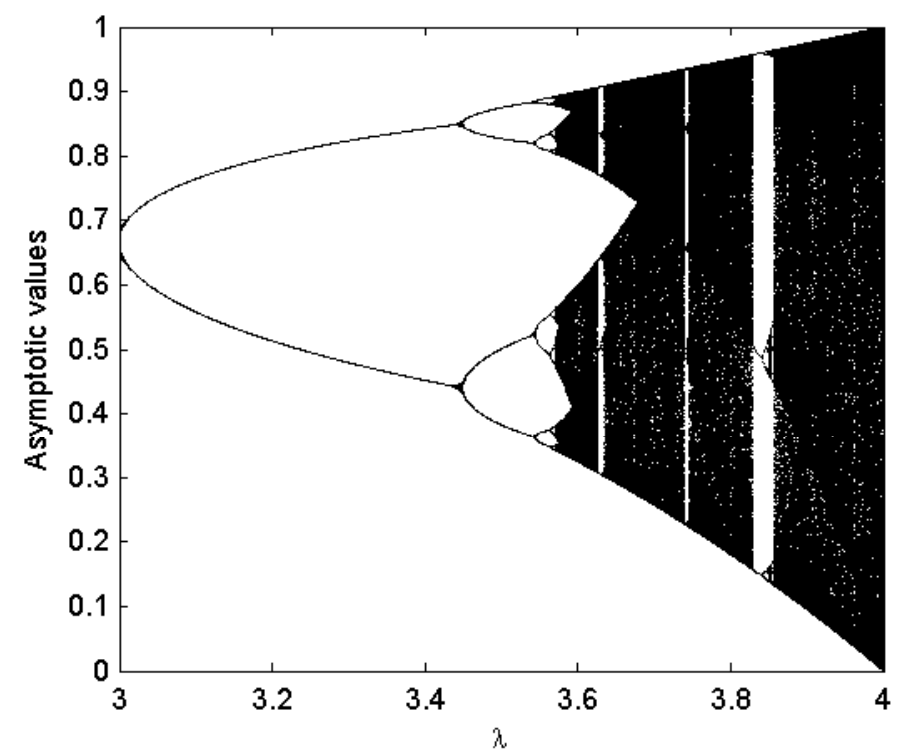

Fig. 1. Bifurcation diagram of the logistic map

where $f_{0}, f_{1}$ are so-called "synthesis filters". To ensure the correct recovery of the plaintext signal, the analysis and synthesis filters must satisfy a certain requirement as shown in Eq. (8) of [12]. The reader is referred to [12] for more information about the inner working of the cryptosystem.

This paper focuses on the security analysis of the above cryptosystem. The next section points out a security problem about the reduction of the key space. Section 3 discusses how to recover the secret key of the cryptosystem by a known-plaintext attack. In the last section the conclusion is given.

\section{Reduction of the key space}

As it is pointed out in [5, Rule 5], the key related to a chaotic cryptosystem should avoid non-chaotic areas. In [12] it is claimed that the key space of the cryptosystem under study is given by the set of values $\lambda_{i}$ and $s_{i}[0]$ satisfying $3<\lambda_{i}<4$ and $0<s_{i}[0]<1$ for $i=0,1$. However, when looking at the bifurcation diagram of the logistic map (Fig. 1), it is obvious that not all candidate values of $\lambda_{i}$ and $s_{i}[0]$ are valid to ensure the chaoticity of the logistic map. There are periodic windows which have to be avoided by carefully choosing $\lambda_{i}$. As a consequence, the available key space is drastically reduced. 


\section{Known-plaintext attack}

In a known-plaintext attack the cryptanalyst possesses a plaintext signal $\{x[n]\}$ and its corresponding encrypted subband signals $\left\{v_{0}[n]\right\}$ and $\left\{v_{1}[n]\right\}$. Because $\left\{h_{0}[n]\right\},\left\{h_{1}[n]\right\}, K_{0}$ and $K_{1}$ are public, we can get $\left\{t_{0}[n]\right\}$ and $\left\{t_{1}[n]\right\}$ from $\{x[n]\}$. Then we can get the values of $\left\{s_{0}[n]\right\}$ and $\left\{s_{1}[n]\right\}$ as follows:

$$
\begin{aligned}
& s_{0}[n]=v_{0}[n]-t_{0}[n]-t_{1}[n], \\
& s_{1}[n]=t_{1}[n]-v_{0}[n]-v_{1}[n] .
\end{aligned}
$$

For $n=0$, the values of the subkeys $s_{0}[0]$ and $s_{1}[0]$ have been obtained. Furthermore, we can obtain the control parameters by just doing the following operations for $i=0,1$ :

$$
\lambda_{i}=\frac{s_{i}[n+1]}{s_{i}[n]\left(1-s_{i}[n]\right)} .
$$

In [12], the authors did not give any discussion about the finite precision about the implementation of the cryptosystem in computers. If the floating-point precision is used, then the value of $\lambda_{i}$ can be estimated very accurately. It was experimentally verified that the error for the estimation of $\lambda_{i}$ using (13), and working with floating-point precision, was never greater that $4 \cdot 10^{-12}$. If the fixed-point precision is adopted, the deviation of the parameter $\lambda_{i}$ estimated exploiting Eq. (13) from the real $\lambda_{i}$ may be very large. Fortunately, according to the following Proposition 1 [13, Proposition 2], the error is limited to $2^{4} / 2^{L}$ (which means only $2^{4}$ possible candidate values to be further guessed) when $s[n+1] \geq 0.5$.

Proposition 1 Assume that the logistic map $s[n+1]=\lambda \cdot s[n] \cdot(1-s[n])$ is iterated with L-bit fixed-point arithmetic and that $s(n+1) \geq 2^{-i}$, where $1 \leq i \leq L$. Then, the following inequality holds: $|\lambda-\tilde{\lambda}| \leq 2^{i+3} / 2^{L}$, where $\tilde{\lambda}=\frac{s[n+1]}{s[n] \cdot(1-s[n])}$.

\section{Conclusion}

In this paper we have analyzed the security properties of the cryptosystem proposed in [12]. It has been shown that there exists a great number of weak keys derived from the fact that the logistic map is not always chaotic. In addition, the cryptosystem is very weak against a known-plaintext attack in the sense that the secret key can be totally recovered using a very short plaintext. 
Consequently, the cryptosystem introduced by [12] should be discarded as a secure way of exchanging information.

\section{Acknowledgments}

The work described in this paper was partially supported by Ministerio de Educación y Ciencia of Spain, Research Grant SEG2004-02418. Shujun Li was supported by the Alexander von Humboldt Foundation, Germany.

\section{References}

[1] S. Li, Analyses and new designs of digital chaotic ciphers, Ph.D. thesis, School of Electronic and Information Engineering, Xi'an Jiaotong University, Xi'an, China, available online at http://www.hooklee.com/pub.html (June 2003).

[2] G. Alvarez, F. Montoya, M. Romera, G. Pastor, Chaotic cryptosystems, in: L. D. Sanson (Ed.), Proc. 33rd Annual 1999 International Carnahan Conference on Security Technology, IEEE, 1999, pp. 332-338.

[3] L. Kocarev, Chaos-based cryptography: A brief overview, IEEE Circuits Syst. Mag. 1 (2001) 6-21.

[4] T. Yang, A survey of chaotic secure communication systems, Int. J. Comp. Cognition 2 (2) (2004) 81-130.

[5] G. Alvarez, S. Li, Some basic cryptographic requirements for chaos-based cryptosystems, International Journal of Bifurcation and Chaos 16 (8) (2006) 2129-2151.

[6] G. Alvarez, F. Montoya, M. Romera, G. Pastor, Cryptanalyzing a discretetime chaos synchronization secure communication system, Chaos, Solitons and Fractals 21 (3) (2004) 689-694.

[7] G. Alvarez, F. Montoya, M. Romera, G. Pastor, Breaking parameter modulated chaotic secure communication system, Chaos, Solitons and Fractals 21 (4) (2004) 783-787.

[8] G. Alvarez, F. Montoya, M. Romera, G. Pastor, Cryptanalyzing an improved security modulated chaotic encryption scheme using ciphertext absolute value, Chaos, Solitons and Fractals 23 (5) (2004) 1749-1756.

[9] G. Alvarez, S. Li, F. Montoya, G. Pastor, M. Romera, Breaking projective chaos synchronization secure communication using filtering and generalized synchronization, Chaos, Solitons and Fractals 24 (3) (2005) 775-783. 
[10] S. Li, G. Alvarez, G. Chen, Breaking a chaos-based secure communication scheme designed by an improved modulation method, Chaos, Solitons and Fractals 25 (1) (2005) 109-120.

[11] G. Alvarez, Security problems with a chaos-based deniable authentication scheme, Chaos, Solitons and Fractals 26 (1) (2005) 7-11.

[12] B. W.-K. Ling, C. Y.-F. Ho, P. K.-S. Tam, Chaotic filter bank for computer cryptography, Chaos, Solitons and Fractals 34 (2007) 817-824.

[13] S. Li, C. Li, G. Chen, K.-T. Lo, Cryptanalysis of the RCES/RSES image encryption scheme, J. Systems and Software, in press, doi:10.1016/j.jss.2007.07.037 (2007). 